\title{
La thérapie assistée par les dauphins : une pratique alternative de soutien à l'interaction
}

Florence Gaunet

\section{(2) OpenEdition}

1 Journals

Édition électronique

URL : http://journals.openedition.org/communicationorganisation/2811

DOI : 10.4000/communicationorganisation.2811

ISSN : $1775-3546$

Éditeur

Presses universitaires de Bordeaux

Édition imprimée

Date de publication : 1 mai 2003

ISSN : 1168-5549

Référence électronique

Florence Gaunet, « La thérapie assistée par les dauphins : une pratique alternative de soutien à l'interaction », Communication et organisation [En ligne], 23 | 2003, mis en ligne le 27 mars 2012, consulté le 19 avril 2019. URL : http://journals.openedition.org/communicationorganisation/2811 ; DOI : 10.4000/communicationorganisation.2811

Ce document a été généré automatiquement le 19 avril 2019

(c) Presses universitaires de Bordeaux 


\title{
La thérapie assistée par les dauphins: une pratique alternative de soutien à l'interaction
}

\author{
Florence Gaunet
}

1 La Thérapie Assistée par les Dauphins - ou TAD - est une pratique thérapeutique psychocorporelle qui s'applique aux enfants et aux adultes présentant des troubles psychomoteurs, cognitifs, de la personnalité et de la communication. Elle vise à initier des changements aux plans attentionnels, du contrôle moteur, de l'humeur, de la communication et de la sociabilité. Elle est fondée sur le développement d'une relation entre un patient et un dauphin et se prolonge, dans les cas de problèmes de personnalité et de communication, avec des procédures de travail sur le développement des relations interindividuelles au sein du groupe de soutien thérapeutique, au sein de la famille et de groupes d'activités sociales.

2 Les premiers travaux en TAD ont commencé dans les années 70 et le premier programme régulier a commencé en 1988, au Dolphin Research Center, en Floride. Les principaux travaux sur l'évaluation des effets de la TAD dans diverses populations de patients, et sur les mécanismes psychologiques, cognitifs et physiologiques à l'origine des effets de la TAD ont été présentés pour la première fois en 1995 et 1996 (Annual International Symposium on Dolphin Assisted Therapy.

Deux points essentiels doivent être soulignés :

- les effets bénéfiques observés peuvent se prolonger jusqu'à au moins un an suivant le traitement;

- la principale modalité d'action de la TAD - et la seule reconnue - repose sur le principe la zoothérapie.

4 Il existe aujourd'hui dans le monde une dizaine de centres de TAD dans lesquels se pratiquent ce que l'on appelle « la nage avec les dauphins ». Dans certain de ces centres, des suivis médicaux et thérapeutiques spécialisés (avec médecins, psychothérapeutes et entraîneurs spécialistes des dauphins) et des recherches scientifiques concernant l'effet 
de la TAD et les processus physiologiques et psychologiques impliqués tant au niveau individuel que systémique, sont pratiqués.

Il est important de préciser que la zoothérapie, la TAD ne bénéficie pas d'une reconnaissance du corps médical et scientifique. Cependant, le développement de la zoothérapie, en général, dans le grand public (équithérapie, introduction des chiens dans les hôpitaux, maisons de retraite, écoles,...) et de la TAD, en particulier, doit conduire le corps médical et scientifique à s'intéresser à cette pratique, tant d'un point de vue des conditions dans lesquelles des effets bénéfiques se développent chez l'Homme que du point de vue de l'étude des processus impliqués lors de la communication non verbale qui s'instaure entre le sujet et l'animal. Le point de l'auteur est de considérer la TAD comme une pratique alternative de soutien thérapeutique permettant l'initialisation de progrès sensori-moteurs et/ou sociaux sur certains types de patients.

\section{Fondement de la Thérapie Assistée par les Dauphins}

6 L'objectif initial et principal de la TAD est de contribuer à la récupération de fonctions mentales d'enfants présentant un retard psychomoteur et psychologique. Il faut également savoir qu'un travail sensori-moteur chez les enfants contribue au développement des capacités de communication (Butterworth et Morissette, 1996). La TAD repose sur le fait que le sujet va accroître son attention du fait de son désir d'interagir avec l'animal. Une procédure de renforcement positif est en outre appliquée au patient afin d'appuyer les comportements cognitifs moteurs et affectifs nouveaux. Le Dr Nathanson - pionnier de la TAD - affirme depuis $1978^{1}$ que la clé de l'amélioration des compétences générales repose sur l'apprentissage et que l'attention est la clé de voûte de l'apprentissage. Le Dr Nathanson a pu montrer que la TAD augmentait significativement les capacités d'apprentissage et de rappel de l'information quand la session d'apprentissage d'enfants atteints du syndrome de Down se faisait avec les dauphins par rapport à une situation d'apprentissage en classe.

7 Le centre du Dr Nathanson a reçu plus de 700 enfants et adultes entre 89 et 96 (site Internet DolphinHumanTherapy), ce qui montre l'intérêt de cette approche. Dans les années 90, les centres de TAD se sont développés en Amérique du nord et du sud. Au ProcheOrient, le centre d'Éclat fait référence, et un centre de nage avec les dauphins en mer associé à des pratiques relevant du domaine du développement personnel ${ }^{2}$ se développe aux Açores.

8 Enfin, depuis moins d'une dizaine d'année, on a pu montrer l'effet de la TAD chez des patients avec des troubles de nature physiologiques (douleur chronique), ce qui a conduit à proposer que les effets de la TAD reposeraient également sur un média autre que psycho-attentionel, de nature physiologique: l'hypothèse est que les émissions acoustiques des dauphins affecteraient la physiologie du patient.

\section{Profil des patients}

9 De façon générale, davantage d'enfants que d'adultes participent à la TAD, principalement du fait que cette pratique suscite davantage d'intérêt pour les enfants que pour les adultes, mais aussi parce que la population des enfants est davantage susceptible que les adultes d'y trouver des bénéfices. La faible participation des adultes résulte 
également du fait que le nombre limité de places disponibles conduit les centres de TAD à favoriser la prise en charge des enfants, car la prise en charge des adultes peut se faire plus facilement par les canaux de la médecine traditionnelle.

On recense une centaine de profils de patients intéressés par la pratique de la TAD (pour un exemple, voir le site DolphinHumanTherapy). Ces profils relèvent des retards de développement sensori-moteur, cognitif et émotionnels, ainsi que d'affections physiques et physiologiques chroniques acquises.

11 Ainsi, on trouvera pour une large part des enfants présentant les troubles suivants: autisme, difficulté émotionnelle et d'estime de soi, déficit attentionnel, syndrome de Down anorexie, dépression, cancer, syndrome de stress post-traumatique dyslexie, retard mental, lésion de la moelle épinière et du cerveau, cécité, surdité, pathologies neuromusculaire, phobie, syndrome de Rett. Les patients cumulent souvent plusieurs handicaps. Chez les adultes, on trouvera essentiellement des patients à tendance dépressive existentielle ou développée à la suite d'une maladie (cancer.) ou d'un accident.

\section{Principe de la Thérapie Assistée par les Dauphins}

12 La TAD se pratique en delphinarium ou bien en environnement marin. Il existe deux procédures qui conduisent à la participation des dauphins: 1) le choix délibéré de l'animal d'interagir avec le patient; 2) le renforcement positif du comportement de l'animal par un thérapeute grâce à l'alimentation, afin de renforcer positivement certaines actions du patient. L'équipe encadrant le patient est généralement constituée d'un psychologue et d'un entraîneur de dauphin. Le rôle du premier est veiller et de contribuer au bon développement des interactions naissantes, et le rôle du second est d'inciter au lien patient-dauphin par des attitudes simples de motivation, facilité par la relation privilégiée préexistante entre l'entraîneur et le dauphin.

Durant la TAD, les difficultés spécifiques du patient sont prises en charge (psychothérapie, rééducation sensori-motrice. communication, langage, etc.). Dans certains centres, la famille participe également à des sessions d'information et d'activités avec le patient: le patient et sa famille peuvent être impliqués dans un ensemble d'activités motivantes, éducatives et de détente.

Les protocoles sont sensiblement similaires d'un centre à l'autre.

Pour les patients ne résidant pas sur place, la TAD se pratique tous les trois mois environ sur quelques années. Dans ce cas. est alors proposé un stage de TAD intensif s'étendant sur une ou deux semaines, à raison d'une séance d'interaction conditionnelle de 20 à 50 minutes par jour et une séance d'interaction libre variant de 20 à 50 minutes par jour.

Pour les résidents, la TAD consiste en général en une session d'une heure par semaine sur la durée d'une année.

17 En fonction du type de pathologie, un programme et des objectifs à atteindre si possible sont préétablis. Par exemple, un objectif dans le cas du soin de l'autisme est de faciliter le contact visuel entre le patient et l'animal, puis entre le patient et l'entraîneur et, enfin, d'étendre progressivement cette capacité à un cercle plus grand d'individus.

Ceci dit, des formules de TAD plus légères, et s'intégrant davantage dans le domaine de développement personnel se développent. Elles n'incluent pas de formules de soutien 
thérapeutique, mais sont associées à des groupes de paroles ou à des activités de relaxation.

\section{Principe d'action de la Thérapie Assistée par les Dauphins} nombreux cas rapportés dans l'ouvrage de B. Belin (2000). Bercovitch (1999) rapporte aussi un très bel exemple de l'effet d'un dauphin sur la récupération de la parole chez un enfant sourd-muet. On note également de façon générale que l'engouement pour ces centres s'est accrue de la part des familles, d'enfants autistes en particulier. De plus, les centres de TAD et les parents d'enfants mentionnent l'amélioration de nombreux comportements (voir les sites Internet des centres de TAD présentés dans la bibliographie ainsi que la section ci-dessous intitulée : «Effet du dauphin sur le patient »).

Cependant, peu d'études scientifiques ont été réalisées afin de valider cette méthode de soutien thérapeutique alternatif. A ce propos. Servais (1999a) rapporte: "Dans un éditorial consacré à la zoothérapie, Rowan (1996) s'étonnait de la discordance entre pratique et recherche dans l'étude des effets thérapeutiques de l'animal. En effet, d'une manière générale, les praticiens observent des résultats positifs alors que, après trente années de recherches, aucun effet bénéfique de l'animal n'a pu être rigoureusement démontré. Il semblerait donc que l'effet thérapeutique de l'animal soit extrêmement sensible à l'appareillage mis en place pour l'évaluer ». Ceci renvoie au problème de l'évaluation scientifique des psychothérapies qui est extrêmement dépendante du contexte thérapeutique (Servais, 1999b) ou, autrement dit, de la relation thérapeutique. Ainsi, si on ôte tout le contexte thérapeutique représenté par la qualité du système interactionnel patient-praticien-support thérapeutique (ici, l'animal ou, ailleurs, une technique psychothérapeutique particulière, i.e. thérapie comportementale et cognitive ou gestalt-thérapie). l'effet positif de la thérapie peut s'en trouver très affecté. Ainsi, Chambon et Marie-Cardine (1999) insistent sur l'importance de la qualité de la relation thérapeutique pour la réussite d'une thérapie. Dans le même sens, on trouve une étude conduite par V. Servais (1999b) : cette chercheuse en anthropologie a "épuré » une situation de TAD jusqu'à contrôler le facteur enthousiasme des thérapeutes : V. Servais a ainsi pu observer la disparition des bénéfices thérapeutiques de la TAD observés initialement, i.e. quand le système interactionnel patient-thérapeuthe-animal se construisait sans contrainte.

Une grande part de l'effet de la TAD reposerait donc sur l'implication forte de l'équipe de soutien thérapeutique et de celle du patient - quand ses moyens mentaux ne sont pas affectés - tous deux empreints du facteur suivant : la fascination par le dauphin. A cet effet. V. Servais (2000) apporte tous les arguments qui conduisent l'homme à se construire une telle représentation. Initialement, ce sont les histoires des rencontres homme-dauphin datant de l'antiquité, encore rapportées aujourd'hui (Bercovitch. 1999), qui conduisent à la «mythification » du dauphin. Puis, depuis plus d'un demi siècle, les études conduites sur leur l'intelligence (capacités perceptives et cognitives, anatomie et physiologie du cerveau) et leur comportement en milieu naturel (migrations, comportements sociaux...) contribuent également à leur attribuer une image 
particulièrement avantageuse et positive. Enfin, les delphinariums. les films d'aventure (Flipper, le dauphin) et documentaires (récemment le phénomène de l'orque Keiko) contribuent également à leur conférer une image fascinante. Cette fascination s'exerce sur l'équipe de soutien thérapeutique qui transmet un enthousiasme certain lors des sessions de soutien; de plus, quand le patient est suffisamment imprégné du monde culturel, cette fascination s'applique directement à lui. Ces facteurs participent fortement à rendre la relation thérapeutique d'une extrême qualité et on a vu précédemment que cette qualité de relation est un facteur fondamental dans l'atténuation des troubles. Dans les cas maintenant où les patients n'ont pas les capacités mentales leur permettant de s'être construit une telle fascination, ce seraient essentiellement les caractéristiques physiques de l'animal qui participeraient à l'effet positif. Ces caractéristiques sont le "sourire » du dauphin, sa grâce, son côté joueur (pour un exemple de thérapie avec le chien, voir Limond et al., 1997), sa peau agréable au toucher et l'harmonie dans les formes et les mouvements de l'animal (une session de TAD utilisant la réalité virtuelle est bénéfique, voir North et al., 1996 et Strickland et al., 1996). En outre, quelles que soient les caractéristiques psychologiques du patient, celui-ci ressent une acceptation et une reconnaissance inconditionnelle lors de l'interaction spontanée et désintéressée avec l'animal ; un support inconditionnel est ainsi fourni à des individus qui n'en reçoivent pas de façon spontanée. Ce facteur semble avoir un poids considérable. Les bénéfices pour le patient lors des sessions de TAD sont de ressentir un moment de joie et de bonheur, un sentiment d'importance et de confiance en soi, un investissement dans une tâche et. enfin, une motivation pour participer, apprendre et réfléchir lorsque les sessions thérapeutiques sont davantage systématisées et contrôlées. Enfin, on sait que le contact avec l'animal est très sécurisant pour l'enfant. Il est reconnu que l'animal représente un objet transitionnel avec le monde extérieur et permet une régression sur laquelle peuvent s'appuyer de nouveaux comportements et donc des progrès d'interaction avec le monde physique et humain (Winnicott, 1971).

23 L'effet de la TAD pourrait également mettre en jeu un second canal: les ondes sonores, voire ultrasonores, émises par les dauphins. De nombreux faits attestent de l'effet positif de certains sons sur le bien-être (voir, dans la bibliographie 'Autres références bibliographiques : musicothérapie, etc.). De plus, les études suivantes ont montré que des modifications physiologiques sont effectivement observables sur les EEG (électroencéphalogrammes). Après le contact avec les dauphins, on observe ainsi une augmentation de la synchronicité entre les deux hémisphères ainsi qu'une augmentation des ondes lentes de type Alpha et Theta (Cole, 1994). Selon Birsh, Cosic et Cole (Birsh et Cosic, 1995 ; Cole, 1995, 1996), une pression ultrasonore induirait des productions de neuromédiateurs dont on observerait les effets grâce aux EEG. Cette hypothèse reste tout à fait spéculative ; cependant elle soulève la question scientifique de l'effet physiologique des ultrasons et des effets répercutés sur le comportement.

Enfin, le troisième canal relève de l'effet de l'hydrothérapie, qui a pour action une relâche des tensions et donc une diminution de l'anxiété. La flottaison facilite la circulation sanguine, réduit la pression sanguine et les pulsations cardiaques. De façon générale, l'immersion aquatique participe de la prise de conscience de son corps, de son propre mouvement. 


\section{Effets du dauphin sur le patient}

Nathanson a pu montrer que des enfants souffrant de lésions cérébrales soumis à une thérapie aquatique voyaient leurs capacités sensori-motrices et cognitives améliorées: l'anxiété, la dépression et la perception de la douleur étaient réduites (Nathanson, 1980. 1989 : Nathanson et al.. 1993, 1997).

Les exemples de résultats obtenus avec la TAD ayant été diffusés à travers le monde sont ceux obtenus à partir des études du Dr Nathanson. En 1980, une étude a testé si l'interaction de deux jeunes enfants atteints du syndrome de Down avec un dauphin améliorait davantage les capacités attentionnelles et langagière que les méthodes conventionnelles (Nathanson, 1980). L'analyse des résultats montre que le petit garçon ne s'exprimant pas verbalement - fournissait davantage de réponses correctes aux tests lorsqu'il interagissait avec le dauphin qu'avec sa mère: la petite fille -s'exprimant verbalement - retenait $13 \%$ de vocabulaire nouveau lorsque le test avait lieu avec l'animal. Pour Nathanson. cette étude montre que le dauphin permet d'augmenter les capacités attentionnelles d'un enfant présentant un certain retard mental.

Plus récemment (Nathanson, 1989). l'apprentissage de mots chez deux groupes distincts de six enfants de 2 à 6 ans (syndrome de Down. hydroencéphalie. lésion cérébrale et multi-handicap) a été comparé. Il a montré que l'apprentissage était dix fois plus important dans le groupe réalisant l'apprentissage en TAD qu'en classe, sur une durée de six mois à raison d'une séance d'apprentissage par semaine. Par ailleurs, le langage de ces enfants et la vitesse de parole se sont améliorés. L'auteur conclut que l'interaction avec les dauphins a facilité les processus attentionnels nécessaires à l'apprentissage.

Plus récemment, Nathanson et de Faria (1993) ont testé l'effet respectif de l'utilisation d'un jouet ou d'un dauphin - en milieu aquatique - comme renforcement de l'apprentissage (4 avait un syndrome de Down, 3 avaient une paralysie cérébrale, 1 avait une lésion cérébrale). On posait des questions générales à l'enfant. Les enfants du second groupe répondaient de façon juste à davantage de questions que le premier groupe.

Puis le groupe du Dr Nathanson (Nathanson et al., 1997) a cherché à déterminer si deux semaines de $\mathrm{TAD}$ pouvait conduire à des améliorations du comportement supérieures à une thérapie physique ou verbale conventionnelle de six mois. Quarante sept enfants ont contribué aux résultats de cette étude. Les capacités motrices et verbales des enfants étaient testées. Les résultats montrent que la TAD permet d'atteindre un meilleur résultat thérapeutique plus rapidement que des thérapies conventionnelles.

Enfin, la question de déterminer dans quelle mesure les améliorations acquises grâce à une session de TAD de une ou deux semaines continuait de faire effet une année après la thérapie (Nathanson, 1998). Les 71 enfants soumis à la TAD avaient les pathologies suivantes: syndrome d'Angelman. syndrome d'Asperger, déficit attentionnel, autisme, paralysie cérébrale, retard de développement, syndrome de Down, hydrocéphalie, microcéphalie, syndrome de Rett, lésion cérébrale traumatique. sclérose... Les parents des enfants ont répondu à questionnaire clinique. L'analyse des réponses fournies par les parents montre que la moitié des améliorations des comportements manifestées pendant la TAD se maintiennent une année après la session de thérapie. Il n'y a pas de différence significative des résultats entre les différentes pathologies testées. Finalement, deux 
semaines de thérapies ont un effet positif supérieur à une semaine de thérapie conventionnelle.

31 Cependant comme le souligne Sophie Donio du Dolphin Reef d'Éclat (Donio et Meir, 1999), dont les propos sont rapportés dans le livre de B. Belin (2000) : « tous les dauphins ne sont pas attirés par les personnes handicapées physiques ou mentales, toutes les personnes handicapées ne sont pas attirées par les dauphins, et quand l'un(e) est attiré par l'autre, et réciproquement, il ne se passe pas automatiquement quelque chose concernant l'évolution de la maladie ou du handicap de la personne ». Enfin, dans la mesure où la TAD procure à l'enfant un soulagement mais ne guérit pas, pour Sophie Donio, il est plus approprié d'appeler la TAD : Expérience de soutien à l'aide de dauphins.

\section{Application de la TAD à l'enfant autiste}

L'autisme touche environ 1 enfant sur 2000. Il se caractérise par une anomalie du développement neuropsychologique portant de façon prédominante sur les fonctions de communication et de socialisation (Bushwick. 2001), mais d'autres fonctions sont également atteintes (Roge, 1999).

De façon générale, l'enfant autiste trouve un grand soulagement lors du contact avec les animaux. L'intérêt de l'usage de l'interaction avec le dauphin repose sur le fait que le dauphin en particulier, recherche l'interaction avec l'enfant autiste et que son approche de l'enfant est particulièrement subtile: le dauphin se laisse facilement toucher par l'enfant. La peau du dauphin étant particulièrement douce et ses mouvements particulièrement bien contrôlés, l'enfant a accès à un contact jugé non agressif. Ce qui lui procure une sensation de douceur et de sécurité particulière. Les angoisses se trouvent donc sensiblement diminuées. Cette sécurisation fait l'objet d'une intervention thérapeutique permettant une régression sur laquelle va s'appuyer un élan vers le monde extérieur. Des interactions avec l'animal s'établissent. Des contacts visuels incidentels puis volontaires s'établissent entre l'enfant et l'animal. Puis l'entraîneur de dauphins entre dans le système relationnel pour former un triangle. Ces nouveaux comportements expérimentés par l'enfant en toute sécurité se transmettent ensuite au système familial. Les premiers apports de la TAD portent donc sur la communication et la socialisation. Le Dr D. Meir, pédopsychiatre à l'Hôpital de Jérusalem, au service des adolescents autistes, considère le dauphin comme un élément transitionnel (au sens de Winnicott. 1971) ouvert par le dauphin. Il a observé des résultats immédiats et durables: les enfants sont plus calmes, montrent moins de comportements stéréotypés, moins d'automutilation. un meilleur contact visuel. Après plusieurs années -le Dr Meir travaille depuis 4 ans avec le centre d'Éclat - on note davantage d'autonomie, une meilleure expression verbale et un meilleur contact avec les enfants ayant eu un soutien thérapeutique avec les dauphins qu'avec ceux qui n'en n'ont pas eu.

De plus, l'intérêt de l'usage de la TAD repose sur l'intérêt fort que l'enfant porte au dauphin. Le dauphin exerce ainsi un pouvoir important de renforcement positif sur l'enfant. Cet outil qu'est le renforcement positif permet à de nombreux comportements déficients d'être mis en place : le pointer du doigt pour désigner un objet de désir, les coordinations sensori-motrices l'apprentissage de la dissociation du Je et du $\mathrm{Tu}$, l'attention conjointe et la représentation des pensées d'autrui (Baron-Cohen, 1991), la stabilisation de son image corporelle (Bullinger. 1998) sur la base non pas du traitement des sensations internes générées par les mouvements de balancements mais par les 
sensations d'origine externes (i.e. utilisation de l'espace extérieur), associer un signe à un acte. etc.

Enfin, les autistes peuvent avoir besoin de nouvelles activités et la TAD est l'une d'elle particulièrement bien acceptée.

\section{Conclusion}

La thérapie assistée par les dauphins apparaît donc comme un outil présentant un grand potentiel de soutien thérapeutique alternatif aux méthodes conventionnelles pour l'amélioration d'un certain nombre de troubles du comportement et d'interaction avec l'environnement physique et relationnel. Elle est très attractive du fait du grand intérêt que suscitent ces animaux pour l'Homme et en particulier pour l'enfant. L'auteur insiste sur le fait que la TAD, au même titre que la zoothérapie, ne bénéficie pas encore de reconnaissance de la part du corps médical et scientifique, que l'état de l'art présenté ici, et les réflexions portées, reposent sur des études ponctuelles et qu'on ne relève quasiment aucune étude publiée dans des revues de psychiatrie, de psychologie ou de sciences du comportement.

Des recherches complémentaires en matière des conditions de validation des effets de la TAD et de la spécification de ces effets sont donc nécessaires. Enfin, l'étude de la relation homme-dauphin, à travers l'étude de leur interaction, voire de leur communication, apparait fondamentale dans le cadre de la compréhension des mécanismes de communication non verbaux et donc dans la construction des organisations.

Dr NATHANSON : http://www.dolphinhumantherapy.com/

S. DONIO : http://www.dolphinreef.co.il/

D. COLE : http://www.aquathought.com/top.html

M. ATLAS : http://www.human-dolphin.org/

\section{BIBLIOGRAPHIE}

BARON-COHEN, S. (1991). The Theory of Mind Déficit in Autism: How Spécific is it? British Journal of Developmental Psychology ; 9, p $301-314$.

BELIN. B. (2000). Animaux au secours du handicap. Paris : L'Harmattan.

BERCOVITCH, P.N. (1999). Oline. le dauphin du miracle. Paris : R. Laffont.

BIRSH, S. \& COSIC, I. (1995). Telemetry Monitoring of Bottlenose Dolphin Biosonar during Human-Dolphin Interaction. First International Symposium on Dolphin Assisted Therapy, Cancun. Mexico, Sept 8-10, abstract. http://www.aquathought.com

BULLINGER. A. (1998). Le dialogue sensori-moteur avec l'enfant : Les particularités de l'enfant à risque autistique. In DELION, P. (Ed) : Les bébés à risques autistiques Paris : Eres, p. 47-59. 
BUSHWICK, N.L. (2001). Social Learning and the Etiology of Autism. New Ideas in Psychology, 19. p. 49-75.

BUTTERWORTH, G. et MORISSETTE. P. (1996). Onset of Pointing and the Acquisition of Language in Infancy. Journal of Reproductive and Infant psychology, 14. p. 219-231.

CHAMBON O. ET MARIE-CARDINE M. (1999) Les hases de la psychothérapie. Dunod. Paris.

COLE, D.M. (1994). Aquathought Foundation Report. Aquathought Fondation, http:// www.aquathought.com

COLE, D. (1995). Neuro-Electrical Effects of Human-Dolphin Interaction and Sono-Chemical Hypotheses. First International Symposium on Dolphin Assisted Therapy, Cancun. Mexico, September. Sept 8-10. abstract. http://www.aquathought.com

COLE, D.M. (1996). Electroencephalographic Results of Human-Dolphin Interaction: A Sonophoresis Model. Second International Symposium on Dolphin Assisted Therapy. Cancun, Mexico, Sept 5-8. abstract. http://www.aquathought.com

DONIO, S. et MEIR, D. (1999). La delphinothérapie. Le Journal des Psychologues, 165, p. 39-43. (Propos recueillis par N. Aymon). LIMOND, J.A., BRADSHAW. J.W.S. et CORMACK, K.F.M. (1997). Behavior of Children with Learning Disabilities lnteracting with a Therapy Dog. Anthrozoôs. 10, p. 84-89.

NATHANSON, D.E. (1980). Dolphins and Kids: A Communication Experiment. Congress proceedings of the XVI world assemhly of the world organisation for preschool éducation, p. 447-451.

NATHANSON. D.E. (1989). Using Atlantic Bottlenose Dolphins to Increase Cognition of Mentally Retarded Children. In P. LOVIBOND et P. W1LSON (Eds.), Clinical and Abnormal Psychology, p. 233-242. Amsterdam: Elsevier, Science Publishers.

NATHANSON. D.E. et DE FARIA, S. (1993). Cognitive Improvement of Children in Water with and Without Dolphins. Anthrozoös, 6, p. 17-29.

NATHANSON, D.E., DE CASTRO, D.. FRIEND. H. et MCMAHON, M. (1997). Effectiveness of ShortTerm Dolphin Assisted Therapy for Children with Severe Disabilities. Anthrozoôs, 10, p. 90-100.

NATHANSON. D. E. (1998). Long-Term Effectiveness of DolphinAssisted Therapy for Children with Severe Disabilities. Anthrozoôs, 11 (1), p. 22-32.

NORTH, M.M.. NORTH. S.M., et COBLE, J.-R. (1996). Virtual Reality Therapy. Colorado Springs : IPI Press.

ROGE, B. (1999). Autisme et autres troubles graves du développement. In HABIMANA. E., TOUSSIGNANT, M. et ETHIER. L.S. (Eds), Manuel de psychiatrie de l'enfant et de l'adolescent. Approche intégrative, p 282-315. Montréal : G. Morin, Ed.

ROWEN, A.N. (1996). Research and Practice. Anthrozoös, 9 (1), p. 2-4.

SERVAIS, V. (1999a). Enquête sur le « pouvoir thérapeutique » des dauphins, ethnographie d'une recherche. Gradhiva, 25, p. 93-105.

SERVAIS, V. (1999b). Some Comments on Context Embodiment in Zootherapy: the Case of the Autidolfijn Project. Anthrozoös, 12, 1, p. 5-15.

STRICKLAND. D., MARCUS, L.M., MESIBOV, G.B. et HOGAN, K. (1996). Brief Report: Two Case Studies Using Virtual Reality as a Learning Tool for Autistic Children. Journal of Autism Development and Disorder, 26, p. 651-659. 
WINN1COTT, D.W. (1975). Objets transitionnels et phénomènes transitionnels. Jeu et réalité. $L$ 'espace potentiel, p. 7-39. Paris : Gallimard.

\section{AUTRES RÉFÉRENCES}

BODSON, V. (1994). Les animaux domestiques et le bien-être des personnes âgées. Mémoire de licence non publié, Université Catholique de Louvain, Louvain-La-Neuve.

HANNICK. S. (1998). « Je ne parlais pas »... Evaluation de l'impact de la communication facilitée sur les comportements-problèmes et l'anxiété des personnes autistes. Mémoire de licence non publié, Université Catholique de Louvain, Louvain-La-Neuve.

LILLY, J. (1978). Communication Between Man and Dolphin. New York: Crown.

O’BARRY, R. (1991). Behind the dolphin smile. New York: Berkley Publishing Group.

POOLE, W. (1993). The Healing Power of Music. In K. BUTTLER et E. FOX (Eds.), The Heart of Healing. p. 130-135. Atlanta : Turner Publishing, Inc.

ROUSSELET-BLANC, V. et MANGEZ, C. (1992). Les animaux guérisseurs. Paris : J.-CI. Lattes.

ROUSSELET-BLANC, V. (1999). Les animaux thérapeutes. Le Journal des Psychologues. 165, p. 36-38.

SITES INTERNET (sous réserve)

S. BOYER : http://www.consciencedauphins.com

\section{NOTES}

1. Nathanson. D.. Dolphin Human Therapy a professional association dedicated to helping disabled children. Dolphin Human Therapj Symposium 1995.

2. Développement personnel : travail sur soi permettant l'atteinte de l'épanouissement personnel et du bien-être.

\section{RÉSUMÉS}

L'article propose une analyse critique des techniques psychocorporelles visant à améliorer les troubles psychomoteurs, cognitifs ou de la communication par le contact avec des dauphins. Le désir d'interaction, soutenu par une forte implication de l'équipe thérapeutique, et éventuellement par des effets biologiques liés aux ondes sonores ou ultrasonores, pourraient expliquer le potentiel d'améliorations constatées chez des enfants autistes, qui devront être confirmées par des études complémentaires. Les mécanismes d'interaction non-verbale entre hommes et dauphins constituent un instrument de compréhension de la communication nonverbale, applicable à la communication dans les organisations.

The article proposes a critical analysis of psycho-corporeal techniques used for the improvement of communication, cognitive. or movement problems through the interaction with dolphins. A strong desire for interaction, an important implication of the therapy team, a possible effects 
linked to sound or ultrasound waves may explain the improvement capacities that can be observed with autistic children. Further complementary studies should confirm these capacities. Non-verbal interaction mechanisms between man and dolphin constitute interesting means of understanding human non-verbal communication, and it's functioning within organizations.

INDEX

Mots-clés : autisme, dauphin, effet thérapeutique, zoothérapie

\section{AUTEUR}

\section{FLORENCE GAUNET}

Florence Gaunet est chercheur au CNRS, en neurosciences comportementalistes, affectée à l'UPR 3251. Ses recherches portent sur l'interaction de l'homme avec son environnement, notamment la cognition spatiale, (gaunet@limsi.fr) 